\title{
Patient preferences for prenatal testing and termination of pregnancy for congenital anomalies and genetic diseases in Ethiopia
}

\section{Daniel Brooks ${ }^{1}$ | Katharine Asta ${ }^{1}$ | Julie Sturza ${ }^{2}$ | Birhanu Kebede ${ }^{3}$ | Delayehu Bekele ${ }^{3}$ | Balkachew Nigatu ${ }^{3}$ । Jenny Hewison ${ }^{4}$ । Shane C. Quinonez ${ }^{5}$ (D)}

\author{
${ }^{1}$ University of Michigan Medical School, Ann \\ Arbor, MI, USA \\ ${ }^{2}$ Department of Pediatrics, University of \\ Michigan Medical School, Ann Arbor, MI, USA \\ ${ }^{3}$ Department of Obstetrics and Gynecology, \\ St. Paul's Hospital Millennium Medical College, \\ Addis Ababa, Ethiopia \\ ${ }^{4}$ Centre for Health Services Research, Leeds \\ Institute of Health Sciences, University of \\ Leeds, Leeds, UK \\ ${ }^{5}$ Department of Pediatrics, Division of \\ Genetics, Metabolism, and Genomic Medicine, \\ University of Michigan, Ann Arbor, MI, USA
}

\section{Correspondence}

Shane C. Quinonez, Department of Pediatrics, Division of Genetics, Metabolism, and Genomic Medicine, University of Michigan, D5240 Medical Professional Building, $1500 \mathrm{E}$. Medical Center Drive, Ann Arbor, MI 48109, USA.

Email: squinon@med.umich.edu

Funding information

Student Biomedical Research Program (SBRP) through the University of Michigan Medical School

\begin{abstract}
Objective: As prenatal diagnostic services expand throughout low-income countries, an important consideration is the appropriateness of these services for patients. In these countries, services now include prenatal ultrasound and occasionally genetic testing. To assess patient interest, we surveyed pregnant patients at a hospital in Addis Ababa, Ethiopia, on their preferences for prenatal testing and termination of affected pregnancies for congenital anomalies and genetic diseases.
\end{abstract}

Method: One hundred one pregnant patients were surveyed on their preferences for prenatal testing and termination of affected pregnancies using a survey covering various congenital anomalies and genetic diseases.

Results: Eighty-nine percent of patients reported interest in testing for all conditions. Three percent of patients were not interested in any testing. Over $60 \%$ of patients reported interest in termination for anencephaly, early infant death, severe intellectual disability, hemoglobinopathy, and amelia. Patients were more likely to express interest in prenatal testing and termination for conditions associated with a shortened lifespan.

Conclusion: Ethiopian patients were interested in prenatal testing and termination of pregnancy for many conditions. Advancing prenatal diagnostic capacities is a potential strategy for addressing the incidence of congenital anomalies and genetic disease in Ethiopia. Importantly, there exist many factors and technological limitations to consider before implementation.

\section{1 | INTRODUCTION}

Over the last two decades, noncommunicable diseases (NCDs) have increased as a cause of worldwide disability and mortality. ${ }^{1,2}$ Concomitant with this increase has been a decrease in disability and mortality from communicable, maternal, neonatal, and nutritional conditions. ${ }^{1}$ This change in disease prevalence mirrors the epidemiological shift countries experience when they improve communicable disease control and the delivery of care to pregnant women, infants, and children resulting in an increase in the relative burden of congenital anomalies and genetic disease. ${ }^{3}$ Low- and middle-income countries (LMICs) are especially susceptible to this transition as they comprise $94 \%$ of all congenital anomalies with genetic or partially genetic origin. ${ }^{4,5}$ As LMICs advance their abilities to treat communicable diseases and to provide peripartum and perinatal care to women and children, respectively, the burden of congenital anomalies and genetic disease steadily increases in incidence and public health importance. $^{3}$ 
To address this changing epidemiology of disease, the World Health Organization has outlined a set of recommendations to address congenital anomalies and genetic disease referred to as Community Genetics. ${ }^{6}$ Community Genetics aims to provide services to individuals while working to reduce the incidence of congenital anomalies and genetic diseases at the population level. ${ }^{6,7}$ Examples of successful programs include those targeting sickle cell disease in Cameroon, Jamaica, Tanzania, and Nigeria and those addressing thalassemias in Bahrain, Iran, and Cyprus. ${ }^{8-14}$ Importantly, each country has a unique set of disorders and cultural and religious beliefs requiring consideration prior to program implementation. ${ }^{3}$

Previous work has evaluated the public's preferences for prenatal testing (PNT) and termination of pregnancies (TOP) for various congenital anomalies and genetic diseases.,15-20 These studies have investigated a range of issues, particularly related to economic, social, and cultural considerations, such as how certain religious values impact preferences for TOP and the influence of gender on attitudes towards PNT based on cultural norms related to child-rearing. ${ }^{8,15-20}$ The majority of these studies have been limited to high- and middleincome countries with the only work performed in a lower middleincome country (Ghana) limited to preferences for sickle cell disease prenatal testing. ${ }^{18}$ Other work has focused on PNT and TOP for select conditions such as hearing loss, familial adenomatous polyposis, and oral clefts. ${ }^{21-24}$ Importantly, no previous work has investigated the broad preferences of pregnant women in a low-income country.

The use of PNT and the termination of those affected with a congenital anomaly and/or genetic disease have been used in many community genetics programs., 5,25 In many low-income countries, the ability to diagnose these conditions is increasing as the result of improving ultrasound diagnosis and prenatal genetic diagnosis. ${ }^{26,27}$ While these advances have the potential to impact a country's incidence of congenital anomalies and genetic disease, there exist multiple ethical, cultural, and legal considerations that must be made prior to the broad introduction of prenatal diagnostic services. ${ }^{3}$ Primary among these considerations are the preferences of pregnant women for PNT and TOP.

In Ethiopia, TOP is important to consider given the recent legal expansion of safe abortion services. ${ }^{28}$ There is limited epidemiological data on genetic conditions or congenital anomalies in Ethiopia with no national standard screening used in pregnancy. ${ }^{29,30}$ However, St. Paul's Hospital Millennium Medical College (SPHMMC) in Addis Ababa, Ethiopia, a low-income country, has recently improved their ability to prenatally screen for and diagnose congenital anomalies and genetic diseases. This has been accomplished through the training of maternal-fetal medicine (MFM) specialists with prenatal ultrasound expertise, genetic counseling education of nurses, and in-country diagnostic genetic testing able to detect pregnancies affected with aneuploidies and select microdeletion/microduplication syndromes. Furthermore, use of a mobile application, the MiGene Family History App, has been used as a tool to introduce medical genetics services while simultaneously collecting epidemiologic congenital anomaly and genetic disease data. ${ }^{30}$ While the treatment of congenital anomalies and genetic diseases is limited in Ethiopia and at SPHMMC,

\section{What's already known about this topic?}

- Prenatal genetic services are expanding throughout many low- and middle-income countries.

- In low- and middle-income countries, little is known on patient preferences for prenatal testing for congenital anomalies and genetic diseases and patient interest in termination of affected pregnancies.

\section{What does this study add?}

- Patients at St. Paul's Hospital in Addis Ababa, Ethiopia, were interested in prenatal testing and termination of pregnancy for many congenital anomalies and genetic diseases.

- Studying patient preferences for genetic services in a low-income country is possible and should be considered prior to the introduction of a new service and/or technology.

there is ongoing capacity building in multiple areas important for the management of these conditions. Examples at SPHMMC include fellowship-trained pediatric specialists important for the care of patients with congenital anomalies and genetic diseases including those in cardiology, neonatology, nephrology, and endocrinology. SPHMMC surgical expertise has also expanded and includes a well-established kidney transplant program.

The introduction of clinical services in Ethiopia has resulted in increased patient access to PNT and the potential for TOP if desired. Increased access to medical care has been shown to drastically improve birth outcomes and women's autonomy in Ethiopia, which is important given their historical lack of decision making and authority. ${ }^{31,32}$ As the introduction of PNT and TOP services requires a significant amount of planning, time, and cost, we aimed to investigate patient preferences for these services at SPHMMC. The information learned from this work may prove useful for various stakeholders including health care providers, public health specialists, and the Ethiopian Ministry of Health as they consider expanding prenatal services into other hospitals and regions. To assess patient preferences for these services, we surveyed pregnant women in the SPHMMC antenatal clinic on their preferences for prenatal testing and TOP for multiple congenital anomalies and genetic diseases.

\section{2 | METHODS}

\section{1 | Survey design}

The patient survey was adapted from that previously designed by Hewison et al. ${ }^{16}$ The survey contained questions with descriptions of 14 congenital anomalies and genetic disorders instead of using medical terminology to avoid preconceived ideas of conditions and 
to describe the conditions to those unfamiliar with medical terminology. The conditions were chosen to reflect a wide range of disorders encompassing physical, mental, and sensory deficits to provide a broad assessment of participants' attitudes. ${ }^{16}$ As an example, a question would be asked as follows: "A child would be born without a brain and die before or soon after birth. Would you want a prenatal test?" "Prenatal test" was used as rapid advancements in genetic testing in Ethiopia that have increased the available testing options, although the current standard of care at SPHMMC is amniocentesis and aneuploidy testing. In a separate question, participants were asked if they would consider TOP if the test showed the fetus had the condition. Answer choices for condition questions were yes, no, and not sure.

As the survey was completed on pregnant women, it also inquired about gestational age, the number of previous pregnancies, participant age, and if a previous pregnancy had resulted in a child born with a congenital anomaly or genetic condition. The survey was available in English and Amharic, the national language of Ethiopia, and was translated independently by two Ethiopian physicians with a consensus version used as the final version.

\section{2 | Patient recruitment}

The study was approved by IRBs at both The University of Michigan and SPHMMC. Consent forms were available in English and Amharic. Participants were recruited from pregnant women awaiting their appointment in the antenatal clinic at SPHMMC. The majority of women approached for participation agreed to participate. Three women chose not to participate but did not provide a reason. SPHMMC is the second largest Ethiopian public hospital and provides most of its care to an underserved population often from surrounding rural areas. Participants were read questions by two SPHMMC Ob/Gyn residents with answers recorded on printed surveys. There was no situation where a man was present when a woman was asked to participate nor during survey administration. Surveys took approximately 5 to 10 minutes per participant. The physicians that facilitated the survey were trained on survey administration including limiting extraneous information outside of the language printed on the survey. If asked a clarifying question, they were trained to repeat the description of the condition as printed on the translated survey.

\section{3 | Statistical analysis}

We used median age (28 years) as a cut point to split responders into two groups for comparison. Fisher exact test was used to compare responses between the two maternal age groups (less than 28 y vs greater than/equal to $28 \mathrm{y}$ ) to examine if there was an age-related difference. Similarly, we used median gestational age (34.5 wk) to split responders into two groups for comparison as this may influence decisions for PNT and TOP. Fisher exact test was also used to compare responses between the two gestational age groups (less than $34.5 \mathrm{wk}$ vs greater than/equal to $34.5 \mathrm{wk}$ ).

Among women who had a previous birth, we compared the responses of the women who had a previous pregnancy that resulted in a child born with a congenital anomaly, congenital anomalies, or a suspected genetic condition $(n=4)$ to those who had not or were unsure ( $n=77$ ) using Fisher exact test. We also assessed group differences based on the number of pregnancies and used three groups for comparison: pregnant once, pregnant twice, and pregnant three or more times using Fisher exact test to compare responses between the three groups.

To determine if individuals were more likely to choose PNT or TOP if conditions were associated with a shortened lifespan, a repeated measures logistic regression was run, modeling responses to PNT or TOP question (yes or no, omitted "not sure" for purposes of this model) as predicted by whether or not conditions included a shortened lifespan. Repeated measures analysis was used to deal with the fact that an individual's responses to all questions would be expected to be correlated, allowing us to remove variability due to individual differences between subjects.

\section{I RESULTS}

\section{1 | Participant characteristics}

A total of 101 participants were enrolled in the study. Table 1 shows the characteristics of participants. The average age of participants was 28 years old ( $n=101$; SD, 4.6; range, 19-37 y) with the average gestational age of 31.9 weeks ( $n=88$; SD, 8.2; range, 7-42 wk) and an average of 2.4 total pregnancies per participant ( $n=101 ; S D, 1.1$; range, $1-5$ previous pregnancies). Of the participants

TABLE 1 Participant characteristics

\begin{tabular}{lc}
\hline & \\
& Mean (range) \\
\hline Patient age & $28(19-37)$ \\
\hline Gestational age & $31.9(7-42)$ \\
\hline Number of pregnancies & $2.4(1-5)$ \\
\hline Previous times pregnant & Number (\%) \\
1 time & $21(21 \%)$ \\
2 times & $40(40 \%)$ \\
3 times & $27(27 \%)$ \\
4 times & $8(8 \%)$ \\
5 or more times & $5(5 \%)$ \\
Previous pregnancy with a birth defect/genetic & $16(75 \%)$ \\
disease & \\
Yes & $4(4 \%)$ \\
No & \\
\hline
\end{tabular}


with a previous pregnancy $(n=80), 5 \%(n=4)$ had a history of a previous pregnancy affected with a congenital anomaly or suspected genetic condition, and $95 \%(n=76)$ had no history of a child affected with a congenital anomaly or genetic condition.

\section{2 | Participants' preferences for prenatal testing}

Figure 1 shows the summary of patient responses regarding preferences for PNT for the 14 conditions surveyed. Of the 101 surveyed patients, $89 \%$ reported wanting testing for all conditions while only $3 \%$ did not want testing for any of the inquired conditions. The only conditions for which respondents were unsure of wanting testing were those describing children with severe intellectual disability, short stature, and behavioral and intellectual disabilities.

In our cohort, the median age of respondents was 28 years. When comparing responses between those less than 28 years and those greater than or equal to 28 years, there was no statistically significant difference in the desire for PNT for any of the inquired conditions. Similarly, the median gestational age was 34.5 weeks with no statistically significant differences noted for PTN for any of the conditions between groups less than 34.5 weeks gestation and those greater than or equal to 34.5 weeks. There was also no difference in preferences for PNT for any of the conditions when comparing respondents who had a previous pregnancy affected with a congenital anomaly $(n=4)$ to those who either were unsure or had not had a previous pregnancy affected with a congenital anomaly ( $n=77$ ). Additionally, no differences were seen for preferences for PNT between groups based on their number of previous pregnancies.

\section{3 | Participants' preferences for termination of pregnancy}

Figure 2 shows the summary of patient responses regarding TOP for the 14 conditions surveyed. Seventeen percent of patients would not consider termination for any of the described conditions. Greater than $60 \%$ of respondents would consider TOP for the following conditions: anencephaly, early infant death, severe intellectual disability, hemoglobinopathy, and amelia. There was no statistically significant difference between age groups regarding interest for TOP for any of the inquired conditions. In terms of gestational age, no difference was seen between those less than 34.5 weeks and

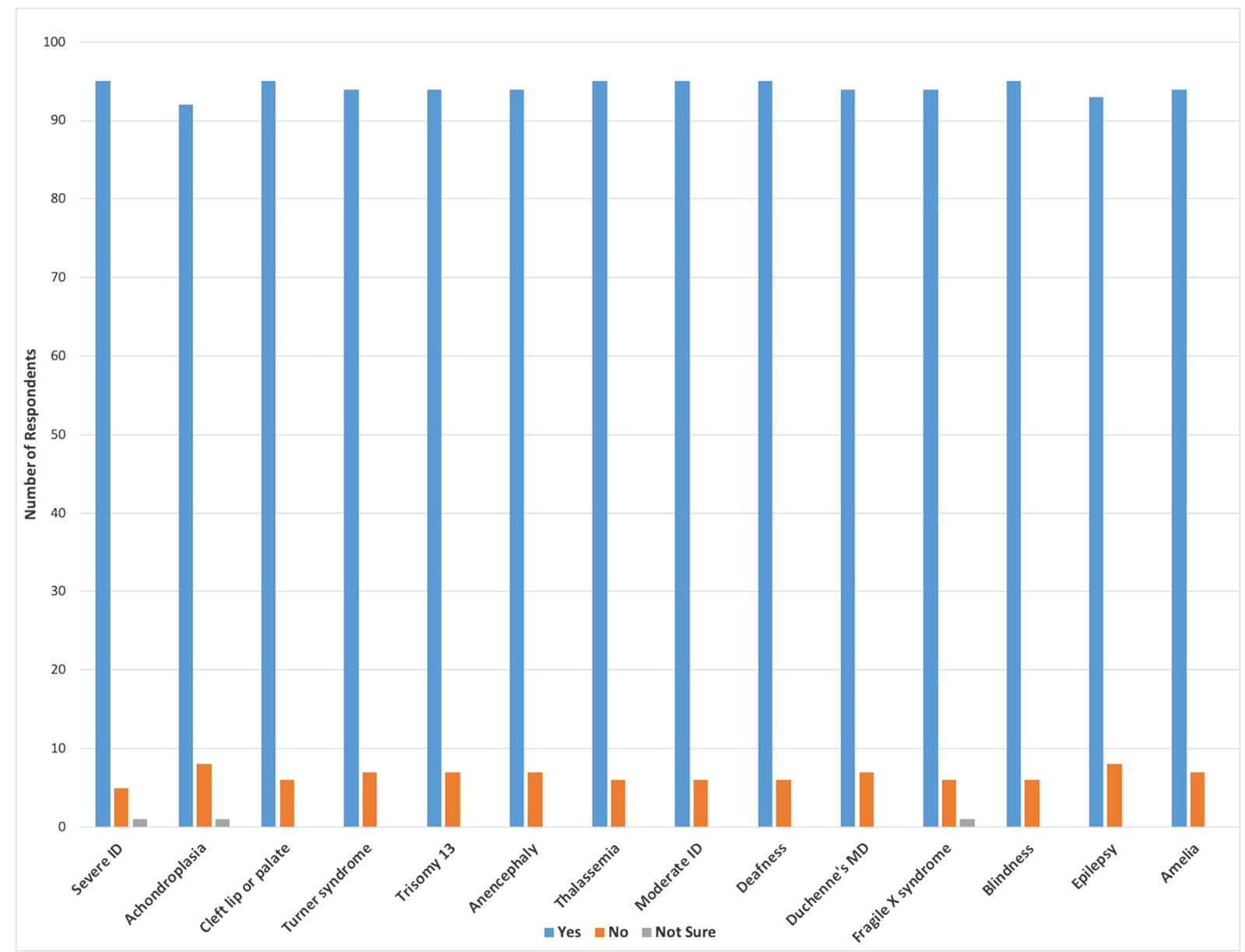

FIGURE 1 Patient preferences for prenatal genetic testing for 14 conditions. ID, intellectual disability; MD, muscular dystrophy [Colour figure can be viewed at wileyonlinelibrary.com] 


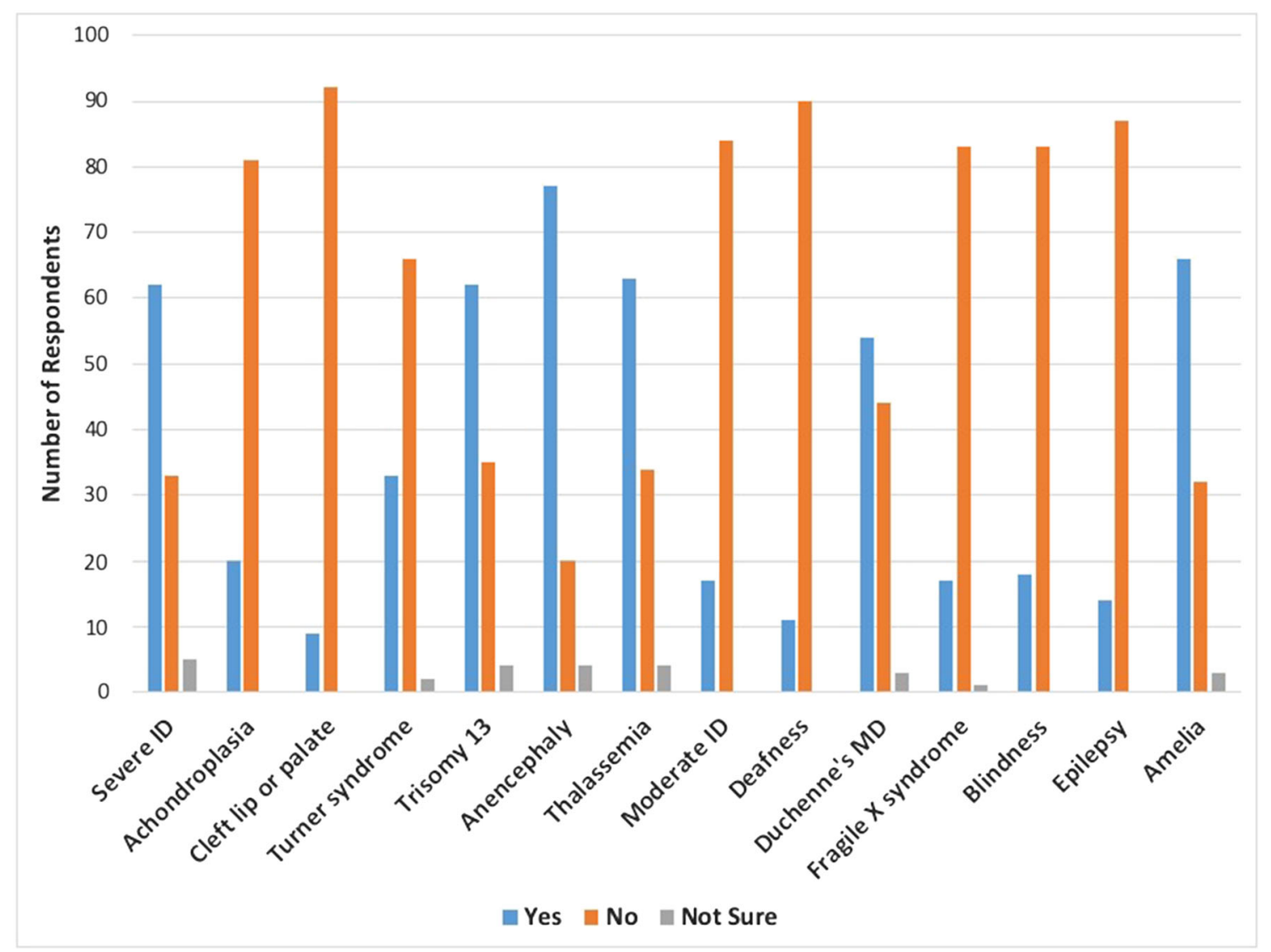

FIGURE 2 Patient preferences for termination of pregnancy for 14 surveyed conditions. ID, intellectual disability; MD, muscular dystrophy [Colour figure can be viewed at wileyonlinelibrary.com]

those greater than or equal to 34.5 weeks for any of the conditions besides blindness. In those with a gestational age greater than or equal to 34.5 weeks, only $6.8 \%$ would consider termination for blindness $(P$ value $=.02)$. When comparing those with a previous pregnancy affected with a congenital anomaly $(n=4)$ to those without a previous pregnancy affected with a congenital anomaly ( $n=77$ ), no difference was seen in their preferences for TOP for any of the conditions.

When comparing groups by number of previous times pregnant, no differences were seen for interest in TOP for all but one of the conditions. For those women with one pregnancy, 14.3\% would consider termination for blindness; for those women with two pregnancies, $7.5 \%$ would consider termination for blindness; and for those women with three or more pregnancies, $30.0 \%$ would consider termination for blindness $(P$ value $=.03$ ). There was also no difference in preferences for TOP for any of the conditions when comparing respondents who had a previous pregnancy affected with a congenital anomaly $(n=4)$ to those who either were unsure or had not had a previous pregnancy affected with a congenital anomaly ( $\mathrm{n}=77$ )

Conditions associated with a shortened lifespan were highly predictive of a "yes" response to desire for PNT or consideration of termination $(P<.0001)$. The odds ratio was 2.60 , meaning the odds of answering "yes" when a condition was associated with a shortened lifespan was 2.60 times the odds of answering "yes" when the condition was not associated with a shortened lifespan.

\section{4 | DISCUSSION}

Here, we show there is broad interest for prenatal genetic services among pregnant women at SPHMMC in Addis Ababa. Most surveyed women were interested in PNT for all queried conditions with women more likely to express interest in TOP for conditions associated with a shortened lifespan. The data presented here for the first time show the potential for prenatal genetic testing and termination of affected pregnancies as a potential tool to address the growing relative incidence of NCDs in low-income countries. This is especially true considering that congenital anomalies are the most common NCD cause of death in individuals less than 5 years of age, with congenital heart defects, neural tube defects, hemoglobinopathies, Down syndrome, and G6PD deficiency, the most common worldwide. ${ }^{4,33}$

To date, there has been limited research focused on prenatal genetic testing in low-income countries. ${ }^{18}$ Ross et al described patient preferences for sickle cell disease prenatal testing in Ghana but did not address TOP of affected pregnancies. ${ }^{18}$ Additional work in Cameroon (a lower middle-income country) also focused on sickle cell disease but 
addressed both PNT and TOP of affected pregnancies. ${ }^{8,34}$ This previous work overall showed patient interest in both PNT and TOP for sickle cell disease. Important to note, most recruited patients had either a personal history of sickle cell disease or a family member with the condition. Here, we assessed the Ethiopian public's preferences for PNT and TOP with a limited number of surveyed individuals having had a child with a congenital anomaly or suspected genetic condition. Even with these differences, there exists similar preferences for PNT between the Ghana and Cameroon studies and our own for conditions surveyed, with similar technological and resource barriers precluding their implementation. 8,18,34

In our cohort, there was broad interest in PNT, which held true based on a number of variables, including age, gestational age, previous number of pregnancies, and having a previous child with a known congenital anomaly. When considering TOP for the same conditions, there was less interest, but women were more likely to express TOP interest for conditions associated with a shortened lifespan. This pattern of responses suggests women are interested in identifying if their child is affected with a condition but may not always be interested in termination, especially if the conditions are not associated with a shortened lifespan. This widespread interest for prenatal testing with less interest in TOP supports the importance of expanding medical genetic services throughout Ethiopia. Patient interest for the early diagnosis of these conditions is best supported through the education and counseling of patients on the many aspects of care for affected individuals. Increasing genetic counseling services in Ethiopia, especially services targeting obstetrical and pediatric populations, would help families understand and adapt to the medical, psychological, and familial implications of the diagnosed condition. As these services are limited in Ethiopia, it will be important to assess if preferences for TOP are different before and after genetic counseling delivery.

For almost all conditions surveyed, there was little variability between the responses in the four different subsamples-age, gestational age, number of pregnancies, and having a previous child with a congenital anomaly. The one exception was blindness in which significantly fewer women above a gestational age of 34.5 weeks would consider TOP. Furthermore, the highest TOP rate for blindness occurred in women with three or more previous pregnancies (30.0\%), while the lowest rate is seen in women who had two pregnancies (7.5\%). The reasons for these responses have not been fully elucidated at this time. Blindness poses a major public health problem in Ethiopia and worldwide with it estimated in low-income countries that $60 \%$ to $80 \%$ of blind children die within 1 to 2 years of becoming blind. ${ }^{35,36}$ Further study is needed to address why these differences were present in our study.

The importance of this work is further evident when considered with related studies in other countries. ${ }^{8,15-21}$ These studies suggest most women desire some form of prenatal testing in both developed and developing nations and most would consider termination for at least some conditions. However, the attitudes towards individual medical conditions differ among various societies and ethnic groups suggesting there may be difficulties with recommending prenatal testing for a universal panel of conditions. Our data support this when considering the increased interest in PNT and TOP for blindness and amelia, both conditions not associated with a significant shortening of lifespan in high-income countries. However, Ahmed et al showed that less than $40 \%$ of British Pakistani mothers were interested in PNT for amelia compared with $93 \%$ of women in our data, illustrating a variation in opinion between groups. ${ }^{15}$

Patients' decision to proceed with TOP is influenced by multiple factors including medical provider recommendations and the personal values and belief systems of the individual and their support network. ${ }^{37}$ In our study, patients were more likely to express interest in TOP for conditions associated with a shortened lifespan. These congenital anomalies and genetic conditions typically entail a large emotional burden and financial cost on the woman and her family, which may be difficult to handle with the already high burden of health care constraints in a developing nation. ${ }^{38}$ As an example, in Ethiopia, patients affected with a neural tube defect were required to wait 447 days for neurosurgical services and had to travel 300 miles on average to receive these services. ${ }^{39}$ Similar difficulties may at least partially explain patients' interest in PNT and TOP for other conditions included in the survey.

While the data presented here will prove useful for future public health planning, such as influencing policy decisions and prioritizing resources for prenatal testing, there are a number of limitations. Our survey was limited to one public institution and did not evaluate ethnicity, religion, education, or socioeconomic background of participants. Furthermore, only a small subset of pregnant women were surveyed. Additionally, the women surveyed had little time to consider their responses and likely had limited knowledge surrounding the conditions. The survey was also overseen by Western researchers, which could have falsely inflated acceptance of prenatal services.

With small numbers of participants in some of the statistical groups, there was limited power to make conclusions in some cases. A potential limitation was that the type of PNT was not specified to account for the full spectrum of PNT available, including ultrasounddiagnosable conditions and those that would require advanced PNT such as certain forms of hearing loss, blindness, and epilepsy. Resultantly, questions were not tailored to current options in Ethiopia.

Future work should include fathers and other family members who have important input when an individual considers PNT and TOP. ${ }^{40,41}$ Additionally, investigating the Ethiopian public's attitudes towards disability and their perception of TOP will be necessary to fully appreciate the implications of PNT and TOP. We chose to assess if patients were more interested in services for conditions associated with a shortened lifespan. There are numerous other factors that contribute to a patient's desire to undergo PNT and TOP that were not assessed here. Future study combined with qualitative data would provide information into these other important condition-specific elements, which may better inform clinical practice. While we used lifespan to assess differences between patient interests in TOP, there are certain to be patient-specific differences in interest. Resultantly, the presence or absence of a condition associated with a shortened lifespan should 
not be used as a primary determinant of whether or not a patient is offered PNT or TOP.

\section{5 | CONCLUSION}

Our work provides preliminary evidence that prenatal diagnostic services and the option for termination is a publicly accepted strategy to address congenital anomalies and genetic diseases in Ethiopia. While it is attractive to consider the potential benefits of PNT and TOP for certain congenital anomalies and genetic conditions, considering their appropriateness for the patient population is paramount.

\section{FUNDING SOURCES}

This work was funded by the Student Biomedical Research Program (SBRP) through the University of Michigan Medical School.

\section{CONFLICTS OF INTEREST}

None declared

\section{ORCID}

Shane C. Quinonez (D) https://orcid.org/0000-0002-8254-0216

\section{REFERENCES}

1. Murray CJL, Lopez AD. Measuring the global burden of disease. N Engl J Med. 2013;369(5):448-457.

2. Hunter DJ, Reddy KS. Noncommunicable diseases. N Engl J Med. 2013;369(14):1336-1343.

3. Christianson A, Modell B. Medical genetics in developing countries. Annu Rev Genomics Hum Genet. 2004;5(1):219-265.

4. Dimes MO. MOD Global Report on Birth Defects January 2006:1-76.

5. Alwan A, Modell B. Recommendations for introducing genetics services in developing countries. Nat Rev Genet. 2003;4(1):61-68.

6. World Health Organization. Community genetics services: report of a WHO consultation on community genetics in low- and middleincome countries. 2011.

7. Kate ten LP, Al-Gazali L, Anand S, et al. Community genetics. Its definition 2010. J Community Genet. 2010;1(1):19-22.

8. Wonkam A, Tekendo CN, Sama DJ, et al. Initiation of a medical genetics service in sub-Saharan Africa: experience of prenatal diagnosis in Cameroon. Eur J Med Genet. 2011;54(4):e399-e404.

9. Makani J, Soka D, Rwezaula S, et al. Health policy for sickle cell disease in Africa: experience from Tanzania on interventions to reduce underfive mortality. Trop Med Int Health: TM \& IH. 2015;20(2):184-187.

10. Grosse SD, Atrash HK, Odame I, Amendah D, Piel FB, Williams TN. The Jamaican historical experience of the impact of educational interventions on sickle cell disease child mortality. Am J Prev Med. 2012; 42(6):e101-e103.

11. Galadanci N, Wudil BJ, Balogun TM, et al. Current sickle cell disease management practices in Nigeria. Int Health. 2014;6(1):23-28.

12. Arrayed Al S. Campaign to control genetic blood diseases in Bahrain. Community Genet. 2005;8(1):52-55.

13. Samavat A, Modell B. Iranian national thalassaemia screening programme. BMJ. 2004;329(7475):1134-1137.
14. Angastiniotis MA, Hadjiminas MG. Prevention of thalassaemia in Cyprus. Lancet. 1981;1(8216):369-371.

15. Ahmed S, Ahmed M, Sharif SM, Sheridan E, Taylor GR. Attitudes towards prenatal testing and termination of pregnancy in British Pakistani parents and relatives of children with recessive conditions in the UK. Prenat Diagn. 2012;32(10):954-959.

16. Hewison J, Green JM, Ahmed S, et al. Attitudes to prenatal testing and termination of pregnancy for fetal abnormality: a comparison of white and Pakistani women in the UK. Prenat Diagn. 2007;27(5): 419-430.

17. Alsulaiman A, Mousa A, Kondkar AA, Abu-Amero KK. Attitudes of Saudi parents with a deaf child towards prenatal diagnosis and termination of pregnancy. Prenat Diagn. 2013;34(2):153-158.

18. Ross PT, Lypson ML, Ursu DC, Everett LA, Rodrigues O, Campbell AD. Attitudes of Ghanaian women toward genetic testing for sickle cell trait. Int J Gynecol Obstet. 2011;115(3):264-268.

19. Arif MO, Fatmi Z, Pardeep B, et al. Attitudes and perceptions about prenatal diagnosis and induced abortion among adults of Pakistani population. Prenat Diagn. 2008;28(12):1149-1155.

20. Souka AP, Michalitsi VD, Skentou $\mathrm{H}$, et al. Attitudes of pregnant women regarding termination of pregnancy for fetal abnormality. Prenat Diagn. 2010;30(10):977-980.

21. Nahar R, Puri RD, Saxena R, Verma IC. Do parental perceptions and motivations towards genetic testing and prenatal diagnosis for deafness vary in different cultures? Am J Med Genet A. 2012; 161(1):76-81.

22. Douma KFL, Aaronson NK, Vasen HFA, Verhoef S, Gundy CM, Bleiker EM. Attitudes toward genetic testing in childhood and reproductive decision-making for familial adenomatous polyposis. Eur J Hum Genet. 2009;18(2):186-193.

23. Wyszynski DF, Perandones C, Bennun RD. Attitudes toward prenatal diagnosis, termination of pregnancy, and reproduction by parents of children with nonsyndromic oral clefts in Argentina. Prenat Diagn. 2003;23(9):722-727.

24. Middleton A, Hewison J, Mueller R. Prenatal diagnosis for inherited deafness-what is the potential demand? J Genet Couns. 2001;10(2): 121-131.

25. Cao A, Rosatelli MC. Screening and prenatal diagnosis of the haemoglobinopathies. Baillieres Clin Haematol. 1993;6(1):263-286.

26. Chandrasekharan S, Minear MA, Hung A, Allyse M. Noninvasive prenatal testing goes global. Sci Transl Med. 2014;6(231): 231fs15-231fs15.

27. Kim ET, Singh K, Moran A, Armbruster D, Kozuki N. Obstetric ultrasound use in low and middle income countries: a narrative review. Reprod Health. 2018;15(1):129.

28. Bridgman-Packer D, Kidanemariam S. The implementation of safe abortion services in Ethiopia. Int J Gynecol Obstet. 2018;143(S4): 19-24.

29. Tadesse L, Tafesse F, Hamamy H. Communities and community genetics in Ethiopia. Pan Afr Med J. 2014;18:115.

30. Quinonez SC, Yeshidinber A, Lourie MA, et al. Introducing medical genetics services in Ethiopia using the MiGene Family History App. Genet Med. 2018;369:448.

31. Tiruneh FN, Chuang K-Y, Chuang Y-C. Women's autonomy and maternal healthcare utilization in Ethiopia. BMC Health Serv Res. 2017; 17(1):1-12.

32. Fantahun $\mathrm{M}$, Berhane $\mathrm{Y}$, Wall $\mathrm{S}$, Byass $\mathrm{P}$, Hogberg $\mathrm{U}$. Womens involvement in household decision-making and strengthening social capitalcrucial factors for child survival in Ethiopia. Acta Paediatr. 2007; 96(4):582-589. 
33. GBD 2015 Child Mortality Collaborators. Global, regional, national, and selected subnational levels of stillbirths, neonatal, infant, and under-5 mortality, 1980-2015: a systematic analysis for the Global Burden of Disease Study 2015. Lancet. 2016;388(10053):1725-1774.

34. Wonkam A, de Vries J, Royal CD, Ramesar R, Angwafo FF III. Would you terminate a pregnancy affected by sickle cell disease? Analysis of views of patients in Cameroon. J Med Ethics. 2014;40(9):615-620.

35. Lewallen S, Courtright P. Blindness in Africa: present situation and future needs. Br J Ophthalmol. 2001;85(8):897-903.

36. Kello AB, Gilbert $\mathrm{C}$. Causes of severe visual impairment and blindness in children in schools for the blind in Ethiopia. $\mathrm{Br} J$ Ophthalmol. 2003;87(5):526-530.

37. Lafarge C, Mitchell K, Fox P. Termination of pregnancy for fetal abnormality: a meta-ethnography of women's experiences. Reprod Health Matters. 2014;22(44):191-201.

38. Chima SC, Mamdoo F. Ethical and legal dilemmas around termination of pregnancy for severe fetal anomalies: a review of two African neonates presenting with ventriculomegaly and holoprosencephaly. Niger J Clin Pract. 2015;18 SuppI(7):S31-S39.
39. Sorri G, Mesfin E. Patterns of neural tube defects at two teaching hospitals in Addis Ababa, Ethiopia a three years retrospective study. Ethiop Med J. 2015;53(3):119-126.

40. Ahmed S, Bryant LD, Tizro Z, Shickle D. Interpretations of informed choice in antenatal screening: a cross-cultural, Q-methodology study. Soc Sci Med. 2012;74(7):997-1004.

41. Schuth W, Karck U, Wilhelm C, Reisch S. Parents' needs after ultrasound diagnosis of a fetal malformation: an empirical deficit analysis. Ultrasound Obstet Gynecol. 1994;4(2):124-129.

How to cite this article: Brooks D, Asta K, Sturza J, et al. Patient preferences for prenatal testing and termination of pregnancy for congenital anomalies and genetic diseases in Ethiopia. Prenatal Diagnosis. 2019;39:595-602. https://doi. org/10.1002/pd.5472 